\title{
A Proposal to Make Biomedical Research into Alzheimer's Disease More Democratic Following an International Survey with Researchers
}

Timothy Daly ${ }^{\mathrm{a}, *}$, Marion Houot ${ }^{\mathrm{b}, \mathrm{c}}$, Anouk Barberousse ${ }^{\mathrm{a}}$, Amélie Petit $^{\mathrm{d}}$ and Stéphane Epelbaum ${ }^{\mathrm{b}, \mathrm{c}}$ a Sorbonne Université, Science Norms Democracy, Paris, France

${ }^{\mathrm{b}}$ Sorbonne Université, Institut du Cerveau - Paris Brain Institute - ICM, Inserm, CNRS, APHP, Hôpital de la Pitié Salpêtrière, Paris, France

${ }^{\mathrm{c}}$ AP-HP, Hôpital de la Pitié Salpêtrière, Institute of Memory and Alzheimer's Disease, Centre of Excellence of Neurodegenerative Disease (CoEN), National Reference Centre for Rare and Early Dementias, Department of Neurology, Paris, France

${ }^{\mathrm{d}}$ Université Paris Saclay, Inserm, CESP, Paris, France

Accepted 5 July 2021

Pre-press 27 July 2021

\begin{abstract}
.
Background: Therapeutic research into Alzheimer's disease (AD) has been dominated by the amyloid cascade hypothesis (ACH) since the 1990s. However, targeting amyloid in AD patients has not yet resulted in highly significant disease-modifying effects. Furthermore, other promising theories of AD etiology exist.

Objective: We sought to directly investigate whether the ACH still dominates the opinions of researchers working on $\mathrm{AD}$ and explore the implications of this question for future directions of research.

Methods: During 2019, we undertook an international survey promoted with the help of the Alzheimer's Association with questions on theories and treatments of AD. Further efforts to promote a similar study in 2021 did not recruit a significant number of participants.

Results: 173 researchers took part in the 2019 survey, 22\% of which held "pro- $\mathrm{ACH}$ " opinions, tended to have more publications, were more likely to be male, and over 60 . Thus, pro-ACH may now be a minority opinion in the field but is nevertheless the hypothesis on which the most clinical trials are based, suggestive of a representation bias. Popular vote of all 173 participants suggested that lifestyle treatments and anti-tau drugs were a source of more therapeutic optimism than anti-amyloid treatments.

Conclusion: We propose a more democratic research structure which increases the likelihood that promising theories are published and funded fairly, promotes a broader scientific view of AD, and reduces the larger community's dependence on a fragile economic model.
\end{abstract}

Keywords: Alzheimer's disease, amyloid- $\beta$, dementia prevention, diversity in science, gender, lifestyle factors, lifestyle interventions, pharmaceutical industry, tau protein, women in science

\footnotetext{
*Correspondence to: Timothy Daly, Science Norms Democracy UMR 8011, 1 Rue Victor Cousin, 75005 Paris, France. E-mail: timothy.daly@paris-sorbonne.fr.
} 


\section{INTRODUCTION}

Disagreement is an obvious fact of science and medicine, but how much is a good thing, and for how long, is worth asking. The community of clinicians and researchers working on Alzheimer's disease (AD) is an amalgam of distinct communities with different approaches to treating cognitive decline in the elderly. The dominant strategy for finding an $\mathrm{AD}$ cure since the 1990s has been targeting AD pathology (amyloid- $\beta(\mathrm{A} \beta$ ) and tau proteins, with $\mathrm{A} \beta$ being the major therapeutic target in our contemporary period (Fig. 1, Liu et al., 2019: "up to 2019 ... the amyloid hypothesis was the most tested $(22.3 \%$ of [human clinical] trials)" [1]). Writing in 2014, Hardy et al. open their paper by claiming that "There is no doubt that for the last 20 years, the $\mathrm{ACH}$ has dominated opinion about the aetiology and pathogenesis of $\mathrm{AD}$, as well as guided the efforts to find treatments" [2]. Nevertheless, there has been a recent shift toward prevention and promotion of resilience to dementia through lifestyle interventions, as well as toward other drug targets, given the uncertainty around the clinical utility of anti-A $\beta$ strategies [1]. Indeed, this shift bears witness to the existence of a variety of promising theories for $\mathrm{AD}$ with compelling evidence in favor of them (two examples being microbes [3] and tau protein initiation [4]).

Scientists are guided in their decision-making by scientific data, but also by opinion. Zollman [5] studied how extreme beliefs and the unequal distribution of information within the research community can lead to "harmful homogeneity in science" (p. 19). The religious language used to describe debates around the suitability of therapeutic targets (defenders of $\mathrm{A} \beta$ and tau proteins as targets being termed BAptists and TAUists, for example [6]), while perhaps used jokingly, is nevertheless suggestive of the possibility of extreme opinions in the AD community. An empirical study of productivity in AD research suggests that "a small percentage of researchers" [7] has access to a large portion of the research apparatus, and while this does not entail that information is distributed unequally, it does suggest the strong

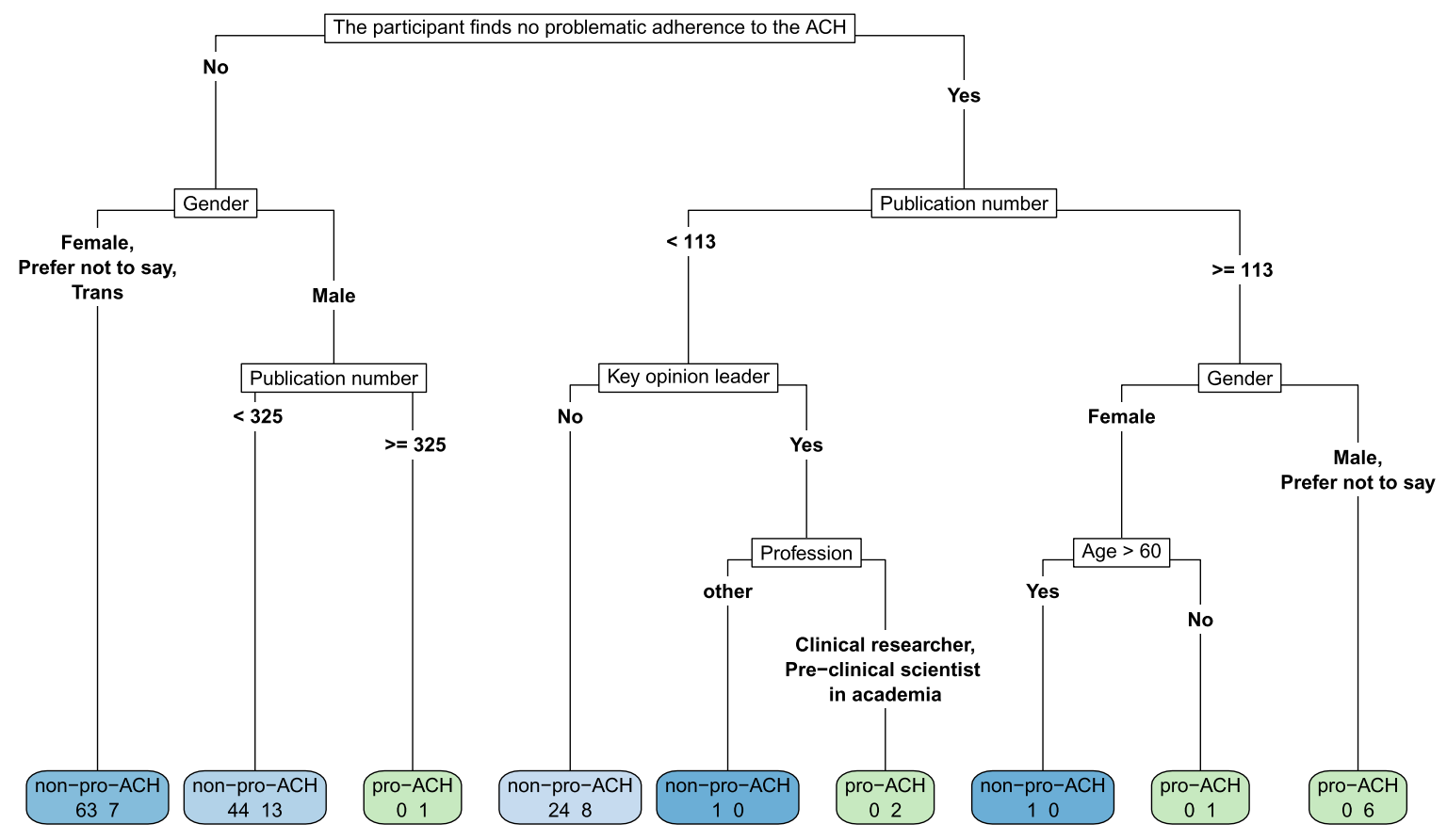

Fig. 1. A decision tree revealing pro-ACH/non-ACH differences according to the participant's view on whether or not there is problematic adherence to the ACH. We cut the depth of the tree to 5. Leave nodes (i.e., the final node, colored in the figure) present the number of pro- $\mathrm{ACH}$ participants on the right and the number of non- $\mathrm{ACH}$ participants on the left. They are light blue to dark blue as a function of the proportion of non- $\mathrm{ACH}$ in the leave node, or they are light green to dark green as a function of the proportion of pro- $\mathrm{ACH}$ in the leave node. In the non- $\mathrm{ACH}$ group, 63 participants (47.37\%) not identifying as male argue that there is problematic adherence to the $\mathrm{ACH}$, compared to only $7(18.42 \%)$ of the pro-ACH group with these characteristics. Conversely, on the other end of the scale, 6 males (or preferred not to say) of the pro-ACH group (15.79\%) argued that there was no problematic adherence to the ACH and had more than 113 median publications. None of the non-ACH had this profile. 
influence of an unrepresentative minority holding a hierarchical sway over the broader direction of the field, at least at the level of publications.

Furthermore, scientific "gatekeeping" in the form of peer review and broader editorial policy offers the advantage of improving the mean quality of published science, yet also increases the risk that more unconventional work is rejected [8]. Indeed, critiques of current funding and publishing models for biomedical research exist beyond the AD field, arguing that conformity to dominant models tends to lead to more funding [9].

These conditions suggest there might well be "harmful homogeneity" in AD research. However, the presence of an influential minority suggests that getting access to most researchers' opinions about AD should not be done via published literature, which cannot adequately represent most researchers working on this disease. We therefore decided to opt for direct access to researchers' opinions about theories and treatments of $\mathrm{AD}$, creating the first anonymous survey into researcher opinions towards theories and treatments of AD. Firstly, we wanted to test Hunter et al. [10]'s hypothesis of "two broad groups; those that support the amyloid cascade hypothesis and those that do not" (p. 254). Secondly, we tested the constituent characteristics of the "pro-ACH" group, before thirdly, looking at possible gender differences in the popular vote toward treatments for AD at different disease stages.

\section{METHODS}

\section{Ethical approval}

The project received ethical approval from the Research Ethics Community of Université Paris Descartes and the data analysis complied with the French Commission nationale de l'informatique et des libertés (CNIL) guidelines. All the data were anonymous and were analyzed in aggregate form. All of the raw survey data are available as Supplementary Material.

\section{Survey design and promotion}

Questions and responses comprised two categories: research (on theories and treatments of $\mathrm{AD}$ ) versus personal (age, profession, country of primary affiliation, clinical versus academic researcher). All questions were optional and multiple choice, based on extensive literature review and consultation with colleagues. The participant filled out the form by following the URL to the Google Forms sheet.

We used Twitter (the account of The Alzheimer's Association International Society to Advance Alzheimer's Research and Treatment "@ISTAART," and T.D.'s personal account, “@PhilAlz”) and a poster at The Alzheimer's Association International Conference (AAIC) 2019 to promote the link to the Google Forms to recruit survey participants between January 1, 2019 and December 31, 2019. A second wave of promotion was undertaken in January 2021, but with less than 20 responders, thus only data from 2019 are analyzed herein.

\section{Hypothesis testing and statistical analysis}

Three hypotheses were tested concerning participant responses. Statistical analyses were performed using R version 3.6.1 (R Foundation for Statistical Computing, Vienna, Austria. URL https://www.Rproject.org/). Participant characteristics were compared between the pro- $\mathrm{ACH}$ and non-ACH groups using Wilcoxon-Mann-Whitney test for continuous variables and Fisher's exact test for categorical variables. Discrepancies in participant response numbers were due to the optional nature of every question.

\section{Hypothesis 1: Pro-ACH responders will account for the majority of the participants}

When participants were asked which categories of drugs gave them hope for a treatment of $\mathrm{AD}$, if they answered "BACE inhibitors" and/or "Anti-A $\beta$ antibodies" (which we combine in Results as " $\mathrm{ACH}$ drugs") and also considered "A $\beta$ physiology (production, clearance, etc.)" to be the number 1 priority in pre-clinical, early, or late stage $\mathrm{AD}$, then they were considered to be "pro-ACH."

\section{Hypothesis 2: The ACH-group will have different constitutive characteristics as compared to the non-ACH group}

In order to describe the profiles of pro- $\mathrm{ACH}$ and non-ACH survey participants, we performed the Classification And Regression Tree (CART) algorithm. The CART algorithm, also known as a "decision tree", is a non-parametric supervised technique that combines variables in such a way as to best discriminate between two groups. We trained a decision tree of depth 5 through entropy minimization with characteristics such as age higher than 60; 
Table 1

Differences in the constitutive characteristics and opinions towards the $\mathrm{ACH}$ of pro-ACH and non-ACH groups identified in the 173 survey participants. Gender differences were significant between the pro-ACH and non- $\mathrm{ACH}$ groups, with significantly more men being pro$\mathrm{ACH}$. Taken together, these results suggest an association between having pro-ACH opinions and more publications, industry money, and self-identifying as a key opinion leader. ${ }^{\ddagger}$ Fisher’s exact test was used to compare groups for categorical variables

\begin{tabular}{|c|c|c|c|c|}
\hline & $\begin{array}{c}\text { All } \\
N=173\end{array}$ & $\begin{array}{c}\text { Non-ACH } \\
\mathrm{N}=133 \\
(76.88 \%)\end{array}$ & $\begin{array}{c}\text { Pro-ACH } \\
\mathrm{N}=38 \\
(21.97 \%)\end{array}$ & $p^{\ddagger}$ \\
\hline Age $>60 y$ & $19(11.05 \%)$ & $10(7.52 \%)$ & $8(21.05 \%)$ & $0.031^{*}$ \\
\hline Gender & & & & $0.035^{*}$ \\
\hline Female & $83(49.70 \%)$ & $71(55.47 \%)$ & $12(31.58 \%)$ & \\
\hline Male & $80(47.90 \%)$ & $54(42.19 \%)$ & $25(65.79 \%)$ & \\
\hline Prefer not to say & $3(1.80 \%)$ & $2(1.56 \%)$ & $1(2.63 \%)$ & \\
\hline Trans & $1(0.60 \%)$ & $1(0.78 \%)$ & $0(0.00 \%)$ & \\
\hline Continent of Major Affiliation & & & & 0.243 \\
\hline North Africa & $1(0.60 \%)$ & $0(0.00 \%)$ & $0(0.00 \%)$ & \\
\hline North America & $101(60.12 \%)$ & $78(60.47 \%)$ & $23(60.53 \%)$ & \\
\hline South America & $11(6.55 \%)$ & $10(7.75 \%)$ & $1(2.63 \%)$ & \\
\hline Asia & $9(5.36 \%)$ & $9(6.98 \%)$ & $0(0.00 \%)$ & \\
\hline Europe & $40(23.81 \%)$ & $28(21.71 \%)$ & $12(31.58 \%)$ & \\
\hline Oceania & $6(3.57 \%)$ & $4(3.10 \%)$ & $2(5.26 \%)$ & \\
\hline Publications number $>100$ & $24(14.04 \%)$ & $14(10.53 \%)$ & $10(27.03 \%)$ & $0.016^{*}$ \\
\hline \multicolumn{5}{|l|}{ Profession } \\
\hline Clinical researcher & $67(38.73 \%)$ & $51(38.35 \%)$ & $15(39.47 \%)$ & 0.236 \\
\hline Other & $18(10.40 \%)$ & $16(12.03 \%)$ & $1(2.63 \%)$ & \\
\hline Pre-clinical scientist in academia & $88(50.87 \%)$ & $66(49.62 \%)$ & $22(57.89 \%)$ & \\
\hline Key Opinion Leader (Yes) & $26(15.48 \%)$ & $16(12.40 \%)$ & $10(26.32 \%)$ & $0.045^{*}$ \\
\hline Received money from pharma company (Yes) & $29(16.86 \%)$ & $18(13.53 \%)$ & $11(28.95 \%)$ & $0.047^{*}$ \\
\hline \multicolumn{5}{|c|}{ Questions regarding the ACH's validity } \\
\hline ACH drugs are NOT a source of optimism for treating human AD. & $86(54.09 \%)$ & $86(71.67 \%)$ & $0(0.00 \%)$ & $<0.001^{*}$ \\
\hline $\begin{array}{l}\text { Beta-amyloid is NOT the \#1 therapeutic priority either at preclinical, } \\
\text { early, or late-stage AD. }\end{array}$ & $119(69.59 \%)$ & $119(90.15 \%)$ & $0(0.00 \%)$ & $<0.001^{*}$ \\
\hline $\begin{array}{l}\text { There is problematic adherence to the ACH from either industry, } \\
\text { academia, associations or funding bodies }\end{array}$ & $125(73.96 \%)$ & $105(80.15 \%)$ & $20(54.05 \%)$ & $0.002^{*}$ \\
\hline Moving forwards (2019-), the ACH is a useful tool to guide research. & $60(35.50 \%)$ & $35(26.92 \%)$ & $24(63.16 \%)$ & $<0.001^{*}$ \\
\hline $\begin{array}{l}\text { Agree with Tanzi (2015): "The clinical trials are failing the hypothesis, } \\
\text { the hypothesis is not failing the trial." }\end{array}$ & $76(44.71 \%)$ & $45(34.35 \%)$ & $30(78.95 \%)$ & $<0.001^{*}$ \\
\hline $\begin{array}{l}\text { Agree with Tanzi (2017): "we need to find people with amyloid buildup } \\
\text { on their brain early" and target it. }\end{array}$ & $89(52.35 \%)$ & $56(42.75 \%)$ & $32(84.21 \%)$ & $<0.001^{*}$ \\
\hline Agree with Davies (2016): “we're flogging a dead horse" (A-beta) & $54(31.76 \%)$ & $52(39.69 \%)$ & $2(5.26 \%)$ & $<0.001^{*}$ \\
\hline $\begin{array}{l}\text { Agree with Herrup (2015): "clinging to an inaccurate disease model is } \\
\text { the option we should fear most." }\end{array}$ & $82(48.52 \%)$ & $75(57.69 \%)$ & $7(18.42 \%)$ & $<0.001^{*}$ \\
\hline
\end{tabular}

gender; country: USA; number of publications; clinical researcher (versus academic); key opinion leader (KOL); has received money from the pharmaceutical industry; whether or not the researcher thinks that "there is problematic adherence to the $\mathrm{ACH}$ from either industry, academia, associations or funding bodies". We used the term adherence so as to insist upon the ACH's ability to guide research.

\section{Hypothesis 3: There will be gender differences in the popular vote towards treatments of $A D$}

We investigated the top three therapeutic targets at pre-clinical, early-stage, and established AD according to popular vote of all the survey participants, pro- $\mathrm{ACH}$ and non- $\mathrm{ACH}$ taken together. Furthermore, if there are gender differences to be found in the pro- $\mathrm{ACH} /$ non- $\mathrm{ACH}$ groups, we might expect to find gender differences in the popular vote. Only participants identifying as $\mathrm{M} / \mathrm{F}$ were included in the gender differences so as to use comparable group sizes for significance testing ( $n=7$ of "trans/prefer not to say/other").

\section{RESULTS}

One hundred and seventy-three participants from across the world filled out the questionnaire, with a median age of 35, 83 (49.7\%) being women. We identified 38 (22.0\%) "pro-ACH" participants, the majority $(65.8 \%)$ of whom were men (Table 1). 
Table 2

The popular vote of all researchers (pro-ACH and non- $\mathrm{ACH}$ taken together) toward therapeutic priorities in AD research, tabulated according to participants' gender. Concerning pharmacological treatments, anti-tau drugs offered more optimism than drug classes inspired by the $\mathrm{ACH}$ (anti-A $\beta$ antibodies and/or BACE inhibitors). The top three therapeutic targets at preclinical, prodromal, and established AD were also investigated. Lifestyle interventions were a top-3 therapeutic priority at all stages of AD. Taken as a whole, the data suggest a favorable opinion regarding lifestyle factors and tau protein intervention. Gender differences in therapeutic priority were only significant for preclinical $\mathrm{AD}$, with significantly more males arguing in favor of anti-A $\beta$ strategies at this stage. ${ }^{\ddagger}$ Fisher’s exact test was used to compare groups for categorical variables

\begin{tabular}{|c|c|c|c|c|}
\hline & $\begin{array}{c}\text { All } \\
\mathrm{N}=173\end{array}$ & $\begin{array}{c}\text { Female } \\
\mathrm{N}=83 \\
(49.70 \%)\end{array}$ & $\begin{array}{c}\text { Male } \\
\mathrm{N}=80 \\
(47.90 \%)\end{array}$ & $p^{\ddagger}$ \\
\hline \multicolumn{5}{|l|}{ Optimism towards the following drugs } \\
\hline Anti-tau & $97(61.01 \%)$ & $50(66.67 \%)$ & $40(53.33 \%)$ & 0.133 \\
\hline Anti-AB antibodies & $62(38.99 \%)$ & $24(32.00 \%)$ & $33(44.00 \%)$ & 0.178 \\
\hline BACE inhibitors & $31(19.50 \%)$ & $15(20.00 \%)$ & $12(16.00 \%)$ & 0.671 \\
\hline \#1 Therapeutic Priority in preclinical AD & & & & $0.020^{*}$ \\
\hline Lifestyle factors (diet, smoking, etc.) & $74(43.53 \%)$ & $39(46.99 \%)$ & $31(39.74 \%)$ & \\
\hline A $\beta$ physiology (production, clearance, etc.) & $33(19.41 \%)$ & $10(12.05 \%)$ & $22(28.21 \%)$ & \\
\hline Inflammation, Microglia, and Astrocytes & $22(12.94 \%)$ & $10(12.05 \%)$ & $11(14.10 \%)$ & \\
\hline \#1 Therapeutic Priority in prodromal AD & & & & 0.060 \\
\hline Lifestyle factors (diet, smoking, etc.) & $49(31.01 \%)$ & $24(32.00 \%)$ & $22(29.73 \%)$ & \\
\hline Tau and NFTs & $40(25.32 \%)$ & $22(29.33 \%)$ & $15(20.27 \%)$ & \\
\hline Inflammation & $26(16.46 \%)$ & $13(17.33 \%)$ & $11(14.86 \%)$ & \\
\hline \#1 Therapeutic Priority in established AD & & & & 0.928 \\
\hline Tau and NFTs & $44(28.21 \%)$ & $19(25.68 \%)$ & $21(28.38 \%)$ & \\
\hline Lifestyle factors (diet, smoking, etc.) & $38(24.36 \%)$ & $20(27.03 \%)$ & $17(22.97 \%)$ & \\
\hline Inflammation, Microglia, and Astrocytes & $29(18.59 \%)$ & $14(18.92 \%)$ & $13(17.57 \%)$ & \\
\hline
\end{tabular}

Pro-ACH participants were more likely to report writing more than 100 publications $(27.0 \%$ versus $10.5 \%$ in the non- $\mathrm{ACH}$ group, $p=0.016$ ), to be a self-reported KOL (26.3\% versuss $12.4 \%, p=0.045)$, to be aged over $60(21.1 \%$ versus $7.5 \%, p=0.031)$, and to have received money from the pharmaceutical industry (29.0\% versus $13.5 \%, p=0.047)$, than non-ACH participants. However, median age group differences did not reach significance. In the non$\mathrm{ACH}$ group, $80.2 \%$ argued that there was problematic adherence to the $\mathrm{ACH}$ from within and outside the scientific community, versus $54.1 \%$ of pro- $\mathrm{ACH}(54.1 \%$ versus $80.2 \%, p=0.002$ ). No difference was found for country or profession.

Concerning the lack of therapeutic progress made in $\mathrm{AD}$ research, $79.0 \%$ of pro- $\mathrm{ACH}$ (versus $34.4 \%$ of non-pro, $p<0.001)$ agreed with Tanzi [11] that "the clinical trials are failing the hypothesis, the hypothesis is not failing the trial." $84.2 \%$ (versus $42.8 \%$, $p<0.001)$ agreed with Tanzi [12] in favor of earlier anti-amyloid strategies in humans, only $5.3 \%$ (versus $39.7 \%$ of non-ACH) agreeing with Davies [13] that such strategies were akin to "flogging a dead horse" when referring to targeting amyloid- $\beta$. As regards the ACH's future, $18.4 \%$ of pro-ACH (versus $57.7 \%$ of non-ACH, $p<0.001)$ agreed with Herrup [14] that "clinging to an inaccurate disease model" was the worst option for the future facing the community.
Finally, in order to better discern pro-ACH versus non-ACH opinions, we used a decision tree (Fig. 1).

We also studied the popular vote of all participants towards treatments and possible gender differences to be found in it. Anti-tau treatments were the highest source of optimism (61.0\% of participants), followed by anti-A $\beta$ antibodies $(39.0 \%)$ and BACE inhibitors (19.5\%). No significant gender differences were found in responses concerning optimism about drug types.

Lifestyle factors were the top therapeutic priority in pre-clinical and prodromal AD (winning $43.5 \%$ and $31.0 \%$ of the popular vote, respectively). In preclinical AD, $\mathrm{A} \beta$ (19.4\%) and inflammation (12.9\%) were the next most popular targets, and in prodromal AD, tau and neurofibrillary tangles (NFTs) $(25.0 \%), \mathrm{A} \beta$ and inflammation (15.2\% and $16.5 \%$ respectively) the next most popular. In established AD, tau and NFTs were the highest therapeutic priority $(28.2 \%$ of the popular vote), followed by lifestyle factors (24.4\%) and inflammation (18.6\%). Concerning gender differences, only at preclinical AD did gender differences reach significance $(p<0.02)$, with men voting comparatively less for lifestyle factors $(39.7 \%$ versus $47.0 \%$ for women), and more for $\mathrm{A} \beta$ (28.2\% versus $12.1 \%$ for women), probably a reflection of the gender division between pro-ACH and non-ACH opinions. 


\section{DISCUSSION}

According to our international survey with 173 participants, pro-ACH opinions did not represent the dominant opinion of researchers working on $\mathrm{AD}$ as of 2019; approximately $22 \%$ of researchers belonged to what we defined as the pro-ACH group. This group tended to argue that the $\mathrm{ACH}$ was a useful tool to guide research, and that there was therapeutic interest in the early targeting of $A \beta$, as opposed to the other "broad group" of researchers [10]. Furthermore, more publication and industrial money is to be found more in the pro-ACH group than in the non- $\mathrm{ACH}$ group.

Nevertheless, the fact that more than half of "pro$\mathrm{ACH}$ " participants agree that there is problematic adherence to the $\mathrm{ACH}$ in the larger community (54\% versus $80 \%$ of non- $\mathrm{ACH}$ ) is consistent with certain researchers that we have interviewed more extensively (TD, AP): they are not ready to let go of the $\mathrm{ACH}$, continue to rely on certain heuristic aspects of it, and at the same time, they are slowly embarking on other paths. This suggests that community-wide movements away from the $\mathrm{ACH}$ are more incremental than revolutionary.

Finally, women were under-represented in the pro$\mathrm{ACH}$ group, representing $32 \%$ of the pro- $\mathrm{ACH}$ and $55 \%$ of the non-ACH group respectively. Concerning gender and age differences, it must not be forgotten that the social structure of biomedical science is hierarchical, with research strategies being mostly directed by principal investigators, i.e., experienced medical doctors and scientists. Differences observed in gender and age may therefore not be related to these variables so much as to the social positions occupied by doctors in the research hierarchy, in which older males are over-represented. Moreover, gender differences themselves may partly have been explained by age, since there were more over 60 s to be found in the group of men (16.3\% versus $4.8 \%)$. Our anecdotal observations (TD, SE) from AlzForum, an influential online community for AD researchers, suggest that the majority of influential commentators on current affairs in $\mathrm{AD}$ research tend to be men in these dominant social positions.

When looking at the popular vote in this survey, anti-tau compounds were a source of greater therapeutic optimism than anti-amyloid strategies, and lifestyle factors were considered to be a top therapeutic priority at all stages of human $\mathrm{AD}$. We will now discuss one way of making $\mathrm{AD}$ research more faithful to popular vote. Nevertheless, before we do so, it is worth noting that there are major limitations to this study.

\section{Study limitations}

Firstly, 173 Twitter-using researchers represent a small minority of $\mathrm{AD}$ researchers (for example, AAIC in Los Angeles in 2019 alone counted 5,700 researchers). And this small sample may have been biased: only those with a strong opinion responding and giving theirs. Thus, the generalizability of our findings may be low. Forcing the research community into polarized groups ("pro-ACH" versus the rest) may not reflect the nuance in opinions that researchers have toward theories which can be studied thanks to other methods, such as bibliometrics [15]. This polarization is aggravated by the fact that quotes taken out of context from the scientific and lay literature (e.g., from Rudolph Tanzi) were used as sources of survey questions.

Concerning self-identification of individuals, our gender categories were highly limited, and our relatively small sample did not allow us to undertake statistical analysis on the contributions of nontraditional or non-conforming gender identities to the popular vote on treatments for AD. It is clear that there is need for greater work on "accountability, justice and representation" for gender minorities in STEM [16]. Furthermore, we did not ask questions on ethnicity, which other STEM researchers are indeed asking so as to "boost diversity in science" [17]. Finally, we did not offer an explicit definition of a "key opinion leader," an ambiguous term whose value to these results is debatable because of the fact we let participants self-identify as KOL or not.

Moreover, as regards the $\mathrm{ACH}$, just as our results suggest that there is some diversity of opinion within the pro-ACH group (e.g., their view of possible problematic adherence to the $\mathrm{ACH}$ ), it is also clear that non-ACH opinions are not of one kind: some researchers are vocally in favor of "rejecting the amyloid cascade hypothesis" [14] and would be more aptly described as "anti-ACH."

Finally, these results are time-sensitive: as different results from clinical trials and other studies are published, so do opinions change toward theories and treatments. The fact that our final round of survey promotion was unsuccessful warrants further analysis into researchers' susceptibility to change their opinion on a scientific topic over a short period of time. The lower participation in 2021 could be due to current events in the field (see Conclusion), 
complications due to the Covid-19 pandemic, or simply the same participant population not wishing to undertake another similar survey. However, any such explanatory hypothesis would be highly speculative, and the issues being discussed in this paper (i.e., the possibility that there might be publishing and funding advantages of supporting the $\mathrm{ACH}$ ) are worthy of further discussion and investigation. Limitations on the speed with which such research can be designed, ethically approved, undertaken, and published, should be taken into account in further studies with similar objectives.

\section{A proposal to make biomedical research into $A D$ more democratic}

It is well-known that biomedical science, as a complex social activity, is guided by non-scientific factors, such as economic interests [18]. Reiss and Kitcher [19] argue that well-ordered biomedical science should follow the "fair-share principle," where the amount of global funds spent on different diseases should be proportional to the suffering caused by them on a global scale. By analogy, we might ask: within the study of a single disease, how should resources best be dedicated to testing hypotheses and developing therapies based on them according to a "fair-share principle"? In other words, how can we make sure that promising theories of AD get their fair share of study and funding?

Solving this incredibly difficult problem is well beyond the scope of this article, but we will offer a sketch of a pro-democracy argument based on "crowd wisdom," the empirical finding that informed collectives outperform individuals in estimating true values of different variables, before underlining two tragedies if the $\mathrm{AD}$ community does not succeed in organizing science better.

Kitcher [20, 21] argues in favor of a democratic deliberation process: taking the points of view of different segments of the community and attempting to guide research according to them. This does not have to mean a majority vote, but the phenomenon known as the wisdom of the crowd [22] suggests that the average value of multiple estimates tends to be more accurate than any one single estimate. Therefore, listening to the popular vote of researchers-at fora such as the yearly AAIC, and pooling a certain percentage of available funding towards the therapeutic leads suggested by popular vote-would mean drawing on many thousands of collective years of experience and perspective, which could lead to more accurate estimates of the causes of $\mathrm{AD}$, and the best treatments to pursue. There is also increasing research being done with dementia patients in a co-research role in gerontology research (for example [23]); there is also much unexplored scope for including the patient community in deliberation processes concerning curative and preventive research into $\mathrm{AD}$, and popular vote could also be used here.

The major idea defended here is that projects should be funded in a way that better represents the plurality of therapeutic leads offered by the research community. A yearly popular vote could be one step in that direction. But this leaves many questions open which we cannot definitively answer in one article, including, but not limited to the following:

How could we improve representation on funding bodies and editorial boards, including a role for the patient community?

Upon what kinds of evidence should publication and funding decisions be based so that both scientific pluralism and plausibility are guaranteed in $A D$ research according to a fair-share principle?

What kind of funding model would be most suited to a more democratic approach: private and/or public ventures?

Are there some domains and methods within biomedical science which might be particularly under the influence of monopolized ways of thinking? (e.g., at the level of pre-clinical or clinical research?) Could publication and funding quotas be used to make monopolized domains more inclusive?

How, and to what extent, could the themes of calls for contributions and projects by publishers and funders be broadened on a long-term, community-wide scale?

Furthermore, a more democratic model itself would not be perfect, particularly if it were taken to the extreme of eroding individual expertise, which is and should remain a cornerstone of rigorous science. Instead, the model we propose serves to reduce monopoly, and thereby take any possible institutionalized brakes off the contributions of individual scientists.

In any case, if research cannot become better organized, we anticipate two major tragedies. The first concerns the survival of the fragile economic model underlying therapeutic research into $\mathrm{AD}$, without which patients will never receive disease-modifying treatment. The second concerns science itself. 
Indeed, the current high-risk model encourages the opposition between patient need and return on investment for innovators. Bringing an AD drug to market is estimated to cost $\$ 5.6$ billion [24]. The developer of the first monopolized disease-modifying treatment of $\mathrm{AD}$ would stand to gain an astronomical return on their major investment. Conversely, when a clinical trial of a much-anticipated AD treatment fails, the market value of the pharmaceutical company that developed it loses as much as $40 \%$ overnight, as in the case of Eli Lilly and solanezumab [13]. In January 2018, the pharmaceutical company Pfizer decided to stop its research on AD and Parkinson's disease by laying off 300 researchers due to numerous drug failures amid a dismal context for research on neurodegenerative diseases: pulling out was part of "an exercise to reallocate spend across our portfolio," according to the company [25]. It is not clear what the future of $\mathrm{AD}$ research looks like, but it is fragile and, in its current state, mostly dependent on amyloid being a viable target, with millions of patients and families living in hope. By ensuring that other promising theories are funded, at a community-wide level, it would allow bets to be hedged against the possibility that the ACH does not deliver on its promises.

The second tragedy, done against science itself and those individuals who defend it, is "epistemic injustice," a term coined by philosopher Miranda Fricker as "wrong done to someone specifically in their capacity as a knower" [26]. Fricker draws on examples from literature and history where factors such as race and gender have led to points of view being ignored and condemned. Within AD research, there are surely examples of intellectually honest researchers defending controversial hypotheses of $\mathrm{AD}$ who have struggled to get data published, receive funding, and retain their place within academia. In other words, certain hypotheses might be rejected not because of scientific argument but rather the social structure of the field of biomedical research. We finish by noting that the results from our small sample tentatively suggest that the majority of women do not support the ACH and may therefore be particularly vulnerable to the negative consequences of a community gatekeeping bias. Taking the example of hypotheses concerning the role of microbes in AD [3], Fig. 1 from Liu et al. (2019) showed that “... up to $2019 \quad \ldots \quad 0.5 \%$ of trials tested the virus hypothesis" [1]. Concerning this "fringe theory ... now, researchers are taking it seriously" [27], but the fact that up to 2019 only 1 in 200 clinical trials were dedicated to testing a direct viral contribution to $\mathrm{AD}$, does beg the question: are theories of $\mathrm{AD}$ being funded according to fair-share principle? Ruth Itzhaki, first author on the previously cited [3] editorial on microbes in $\mathrm{AD}$, has described "a series of battles ... awful problems getting [research] published" [28]. This example does point to the possibility of epistemic injustice in $\mathrm{AD}$ research and suggests the existence of perspectives whose contribution to improving the lives of AD patients has not yet been fully taken into account. This seems like community-wide oversight, since the perspectives of marginalized individuals in institutionalized social structures may offer particularly insightful contributions to research, since they may recognize patterns in the world that those in more dominant groups may be blinded to [29].

\section{CONCLUSION}

The recent, controversial accelerated FDA approval of Biogen/Eisai's Aducanumab for use in mild $\mathrm{AD}$ is a testament to the influence of the $\mathrm{ACH}$ and its defenders on the scientific and wider community. The tentative results found in our survey suggest that there is a complex scientific landscape behind the scenes which risks becoming even more polarized following such divisive decision-making [30]. Given the hardships of the research community in finding a disease-modifying treatment for $\mathrm{AD}$, we argue that further efforts should be made to explore democratic solutions to overcome research monopolies so that their potential consequences for patients and scientists can be reduced, and clinically useful treatments for AD be found as soon as possible. It appears that the optimism toward the ACH which has motivated industry and the recent FDA decision may well not be shared by the majority of researchers working on AD. This study offers one tool to study this otherwise silent majority, whose collective wisdom, we argue, could and should be taken into further consideration for the future of vital research into this devastating, complex disease.

\section{ACKNOWLEDGMENTS}

The authors thank the Alzheimer's Association for their invaluable help promoting the survey, Yves Agid and Marc Dhenain for help with formulating some survey questions, and three anonymous reviewers at JAD Reports who all provided helpful feedback. TD thanks the Fondation Médéric Alzheimer for financial 
support from his doctoral bursary 2019-2021, Cédric Paternotte for his inspiring Master's classes on social epistemology, and Pedro Lippmann for the Miranda Fricker reference.

\section{CONFLICT OF INTEREST}

Timothy Daly, Marion Houot, Anouk Barberousse, and Amélie Petit declare no conflicts of interest. Stéphane Epelbaum has received consulting fees from Biogen, Roche, Eisai and GE Healthcare.

\section{SUPPLEMENTARY MATERIAL}

The supplementary material is available in the electronic version of this article: https://dx.doi.org/ 10.3233/ADR-210030.

\section{REFERENCES}

[1] Liu PP, Xie Y, Meng XY, Kang JS (2019) History and progress of hypotheses and clinical trials for Alzheimer's disease. Signal Transduct Target Ther 4, 29.

[2] Hardy J, Bogdanovic N, Winblad B, Portelius E, Andreasen N, Cedazo-Minguez A, Zetterberg H (2014) Pathways to Alzheimer's disease. J Intern Med 275, 296-303.

[3] Itzhaki RF, Lathe R, Balin BJ, Ball MJ, Bearer EL, Braak H, Bullido MJ, Carter C, Clerici M, Cosby SL, Del Tredici K, Field H, Fulop T, Grassi C, Griffin WS, Haas J, Hudson AP, Kamer AR, Kell DB, Licastro F, Letenneur L, Lövheim H, Mancuso R, Miklossy J, Otth C, Palamara AT, Perry G, Preston C, Pretorius E, Strandberg T, Tabet N, TaylorRobinson SD, Whittum-Hudson JA (2016) Microbes and Alzheimer's disease. J Alzheimers Dis 51, 979-984.

[4] Arnsten AFT, Datta D, Tredici KD, Braak H (2021) Hypothesis: Tau pathology is an initiating factor in sporadic Alzheimer's disease. Alzheimers Dement 17, 115-124.

[5] Zollman K (2010) The epistemic benefit of transient diversity. Erkenntnis 72, 17-35.

[6] Mudher A, Lovestone S (2002) Alzheimer's disease - do tauists and baptists finally shake hands? Trends Neurosci 25, 22-26.

[7] Sorensen AA (2009) Alzheimer's disease research: Scientific productivity and impact of the top 100 investigators in the field. J Alzheimers Dis 16, 451-465.

[8] Siler K, Lee K, Bero L (2015) Measuring the effectiveness of scientific gatekeeping. Proc Natl Acad Sci U S A 112, 360-365.

[9] Nicholson JM, Ioannidis JP (2012) Research grants: Conform and be funded. Nature 492, 34-36.

[10] Hunter S, Friedland RP, Brayne C (2010) Time for a change in the research paradigm for Alzheimer's disease: The value of a chaotic matrix modeling approach. CNS Neurosci Ther 16, 254-262.
[11] Tanzi R. 'New clarity' against Alzheimer's. In Harvard Gazette, URL https://news.harvard.edu/gazette/story/ 2015/05/new-clarity-against-alzheimers/. Posted May 5, 2015. Accessed May 3, 2021.

[12] Tanzi R. One doctor's hopeful plan to eradicate Alzheimer's. In Forbes, editor. 2017. https://www.forbes.com/sites/robin seatonjefferson/2017/06/21/new-drug-that-could-helperadicate-alzheimers-is-ready-for-trials/?sh=1539700564 96. Posted June 21, 2017. Accessed May 3, 2021.

[13] Abbott A, Dolgin E (2016) Failed Alzheimer's trial does not kill leading theory of disease. Nature 540, 15-16.

[14] Herrup K (2015) The case for rejecting the amyloid cascade hypothesis. Nat Neurosci 18, 794-799.

[15] Daly T, Houot M, Barberousse A, Agid Y, Epelbaum S (2020) Amyloid- $\beta$ in Alzheimer's disease: A study of citation practices of the amyloid cascade hypothesis between 1992 and 2019. J Alzheimers Dis 74, 1309-1317.

[16] Aguilar R (2021) Breaking the binary by coming out as a trans scientist. Nature 591, 334-335.

[17] Ortega RP (2021) Black scientists gather to form communities and boost diversity in science. Nat Med 27, 756-758.

[18] Pogge T (2005) Human rights and global health: A research program. Metaphilosophy 36, 182-209.

[19] Reiss J, Kitcher P (2009) Biomedical research, neglected diseases, and well-ordered science. THEORIA 24, 263-282.

[20] Kitcher P (2009) Science, Truth, and Democracy, Oxford University Press, Oxford, England.

[21] Kitcher P (2011) Science in a Democratic Society, Prometheus, Buffalo, NY.

[22] Surowiecki J (2004) The Wisdom of Crowds: Why the Many Are Smarter Than the Few and How Collective Wisdom Shapes Business, Economies, Societies and Nations, Doubleday, New York City.

[23] Harding AJE, Morbey H, Ahmed F, Opdebeeck C, Elvish R, Leroi I, Williamson PR, Keady J, Reilly S (2020) Core outcome set for nonpharmacological communitybased interventions for people living with dementia at home: A systematic review of outcome measurement instruments. Gerontologist, doi: 10.1093/geront/gnaa071

[24] Scott TJ, O'Connor AC, Link AN, Beaulieu TJ (2014) Economic analysis of opportunities to accelerate Alzheimer's disease research and development. Ann N Y Acad Sci 1313, 17-34.

[25] Hawkes N (2018) Pfizer abandons research into Alzheimer's and Parkinson's diseases. BMJ 360, k122.

[26] Fricker M (2009) Epistemic Injustice: Power and the Ethics of Knowing. Oxford University Press, Oxford.

[27] Abbott A (2020) Are infections seeding some cases of Alzheimer's disease? Nature 587, 22-25.

[28] Makin S (2018) The amyloid hypothesis on trial. Nature 559, S4-S7.

[29] Allen BJ (1996) Feminist standpoint theory: A black woman's (re) view of organizational socialization. Commun Stud 47, 257-271.

[30] Fleck LM (2021) Alzheimer's and Aducanumab: Unjust profits and false hopes. Hastings Cent Rep 51, 9-11. 